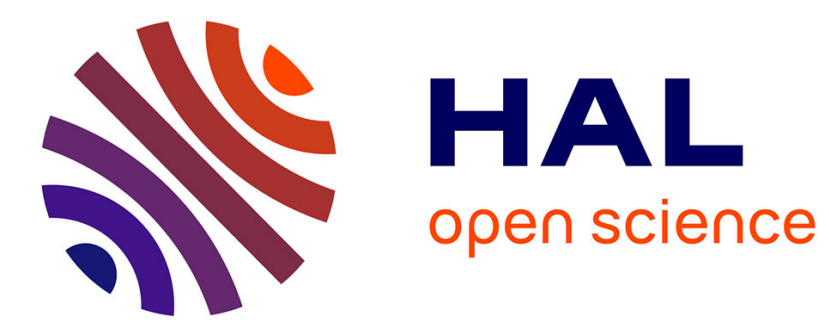

\title{
Portfolio optimization under model uncertainty and BSDE games
}

\author{
Bernt Oksendal, Agnès Sulem
}

\section{To cite this version:}

Bernt Oksendal, Agnès Sulem. Portfolio optimization under model uncertainty and BSDE games.

[Research Report] RR-7554, INRIA. 2011, pp.23. inria-00570532

\section{HAL Id: inria-00570532 \\ https://hal.inria.fr/inria-00570532}

Submitted on 28 Feb 2011

HAL is a multi-disciplinary open access archive for the deposit and dissemination of scientific research documents, whether they are published or not. The documents may come from teaching and research institutions in France or abroad, or from public or private research centers.
L'archive ouverte pluridisciplinaire HAL, est destinée au dépôt et à la diffusion de documents scientifiques de niveau recherche, publiés ou non, émanant des établissements d'enseignement et de recherche français ou étrangers, des laboratoires publics ou privés. 


\title{
Portfolio optimization under model uncertainty and BSDE games
}

\author{
Bernt Øksendal — Agnès Sulem
}

\author{
$\mathbf{N}^{\circ} \mathbf{7 5 5 4}$ \\ March 2011
}

Stochastic Methods and Models

\section{apport}

de recherche 



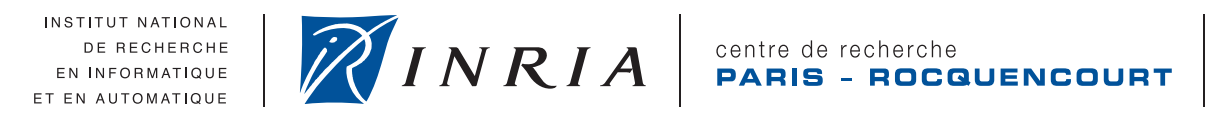

\title{
Portfolio optimization under model uncertainty and BSDE games
}

\author{
Bernt Øksenda: \\ Theme : Stochastic Methods and Models \\ Équipe-Projet Mathfi \\ Rapport de recherche $\mathrm{n}^{\circ} 7554$ - March 2011 -20 pages
}

\begin{abstract}
We consider some robust optimal portfolio problems for markets modeled by (possibly non-Markovian) jump diffusions. Mathematically the situation can be described as a stochastic differential game, where one of the players (the agent) is trying to find the portfolio which maximizes the utility of her terminal wealth, while the other player ("the market") is controlling some of the unknown parameters of the market (e.g. the underlying probability measure, representing a model uncertainty problem) and is trying to minimize this maximal utility of the agent. This leads to a worst case scenario control problem for the agent.

In the Markovian case such problems can be studied using the Hamilton-Jacobi-Bellman-Isaacs (HJBI) equation, but these methods do not work in the non-Markovian case. We approach the problem by transforming it to a stochastic differential game for backward differential equations (BSDE game). Using comparison theorems for BSDEs with jumps we arrive at criteria for the solution of such games, in the form of a kind of non-Markovian analogue of the HJBI equation. The results are illustrated by examples.
\end{abstract}

Key-words: model uncertainty, portfolio optimization, exponential utility, BSDEs, stochastic differential games, Itô -Lévy processes

MSC2010: 91G80, 93E20, 91A23, 60H20, 60H75

* Center of Mathematics for Applications (CMA), Dept. of Mathematics, University of Oslo, P.O. Box 1053 Blindern, N-0316 Oslo, Norway, email: oksendal@math.uio.no and Norwegian School of Economics and Business Administration, Helleveien 30, N-5045 Bergen, Norway. The research leading to these results has received funding from the European Research Council under the European Community's Seventh Framework Programme (FP7/2007-2013) / ERC grant agreement no [228087]

$\dagger$ INRIA Paris-Rocquencourt, Domaine de Voluceau, Rocquencourt, BP 105, Le Chesnay Cedex, 78153, France, email: agnes.sulem@inria.fr 


\section{Optimisation de portefeuilles avec incertitude de modèle et jeux différentiels stochastiques}

Résumé : On considère des problèmes d'optimisation de portefeuilles dans des marchés avec sauts non markoviens. Ces problèmes peuvent être modélisés comme des jeux différentiels stochastiques à 2 joueurs: l'un des joueurs (l'investisseur) essaie de déterminer la stratégie d'investissement qui maximise une fonction d'utilité de sa richesse terminale, alors que l'autre joueur ("le marché") contrôle des paramètres inconnus du marché, à savoir la probabilité sous-jacente représentant l'incertitude de modèle, et cherche à minimiser l'utilité maximale de l'agent. Cela conduit à un problème de contrôle de type pire des cas pour l'investisseur. Dans le cas Markovien, ces problèmes peuvent être étudiés au moyen de l'équation d'Hamilton-Jacobi-Bellman-Isaacs (HJBI). Dans le cas non Markovian, on transforme le problème en un jeux différentiel stochastique pour équations différentielles stochastiques rétrogrades (EDRS). En utilisant des théorèmes de comparaison pour les EDSR avec sauts, on obtient un critère pour la solution de ces jeux, qui peut etre vue comme l'analogue non markovienne de l'équation d'HJBI. Les résultats sont illustrés par des exemples.

Mots-clés : incertitude de modèle, Optimisation de portefeuilles, équations différentielles stochastiques rétrogrades, jeux différentiels stochastiques, processus d'Itô -Lévy 


\section{Introduction}

The financial crisis has led to an increased interest in the role of mathematical models in finance. In particular, it has been pointed out that model uncertainty should be taken into account more often. One way to present model uncertanty mathematically, is by means of a family $\mathcal{Q}$ of probability measures $Q$ which are equivalent to the original probability measure $P$, and by allowing uncertainty regarding which of the measures $Q$ should be taken into account when evaluating performance.

For example, a cautious agent might prefer to trade under the worst case scenario assumption, i.e. to trade optimally being prepared for the worst possible choice of $Q$. Mathematically this leads to a stochastic differential games between the agent, choosing the portfolio, and the "market", choosing the "scenario" measure $Q$.

It is the purpose of this paper to study general non-Markovian stochastic differential games in a market where the stock price is represented by an Itô-Lévy process.

In the Markovian case such games can be studied by using dynamic programming and the Hamilton-JacobiBellman-Isaacs (HJBI) equation. See e.g. 14] and [15].

However, no similar solution method seems to have been available in the non-Markovian case. We approach the problem by transforming it to a stochastic differential game for backward differential equations (BSDE game). Although the relation between stochastic control and BSDEs is well known (see e.g. Chapter 7 of [20] and the recent paper [11), the application to stochastic differential games is new. Using comparison theorems for BSDEs withh jumps we arrive at tractable criteria for the solution of such games, in the form of a kind of non-Markovian analogue of the HJBI equation (Theorem 3.1).

In Section 2 we derive the basic general relation between the optimal portfolio problem and the associated BSDE. We consider 3 types of utility functions: Exponential utility, power utility and logarithmic utility. Another method is proposed for general utility functions. In Section 3 we apply the results of Section 2 to obtain our main BSDE games verification theorem, Theorem 3.1] Then we apply this to study specific optimal portfolio problems under model uncertainty (worst case scenario).

\section{A BSDE approach to optimal control of Itô-Lévy processes}

Let $X^{u}(t)=X_{x}^{u}(t)$ be a controlled Itô Lévy process on a probability space $\left(\Omega, \mathcal{F},\left(\mathcal{F}_{t}\right)_{t \geq 0}, P\right)$ of the form

$$
\begin{aligned}
d X^{u}(t)=b( & t, u(t), \omega) d t+\sigma(t, u(t), \omega) d B(t) \\
& +\int_{\mathbb{R}} \gamma(t, u(t), z, \omega) \tilde{N}(d t, d z) ; 0 \leq t \leq T \\
X^{u}(0)=x & \in \mathbb{R}
\end{aligned}
$$

where $B$ is Brownian motion, and $\tilde{N}(d t, d z)=N(d t, d z)-\nu(d z) d t$ is the compensated jump measure where $\nu$ is the measure of a Lévy process $\eta$ with jump measure $N$ such that $E\left[\eta_{t}^{2}\right]<\infty$ for all $t$. For simplicity we assume that $b(t, u(t), \omega), \sigma(t, u(t), \omega)$ and $\gamma(t, u(t), z, \omega)$ are given bounded predictable processes for each control process $u$.

For a given initial time $t$ and initial state $x$, we denote by $X_{t, x}^{u}(s)$ the associated process, $0 \leq t \leq s \leq T$.

We want to maximize a performance functional of the type

$$
J^{u}(t)=E\left[U\left(X_{t, x}^{u}(T)+F\right) \mid \mathcal{F}_{t}\right], \quad t \in[0, T] ; \quad u \in \mathcal{A},
$$

where $U: \mathbb{R} \rightarrow \mathbb{R}$ is a given utility function, $\mathcal{A}$ is a given family of admissible $\mathcal{F}_{t}$-adapted controls $u(\cdot)$, and $F$ is a given bounded $\mathcal{F}_{T}$-measurable random variable. Note that $J^{u}(T)=U(x+F)$.

$\mathrm{RR} \mathrm{n}^{\circ} 7554$ 


\subsection{The exponential utility case}

The method described in this section is basically well known albeit maybe not in the general context of Itô Lévy processes (see in particular [1]). For completeness we give a detailed exposition below.

We consider here the performance functional of exponential utility type i.e. we choose

$$
U(x)=-\exp (-\alpha x) ; \quad x \in \mathbb{R}, \alpha>0 \text { constant. }
$$

This gives

$$
J^{u}(t)=-E\left[\exp \left(-\alpha X_{t, x}^{u}(T)-\alpha F\right) \mid \mathcal{F}_{t}\right] ; t \in[0, T]
$$

and since

$$
X_{t, x}^{u}(T)=X_{0, x}^{u}(T)-X_{0,0}^{u}(t)
$$

we can write

$$
J^{u}(t)=M^{u}(t) y^{u}(t)
$$

where

$$
\begin{aligned}
M^{u}(t) & =-E\left[\exp \left(-\alpha X_{0, x}^{u}(T)-\alpha F\right) \mid \mathcal{F}_{t}\right], \\
y^{u}(t) & =\exp \left(\alpha X_{0,0}^{u}(t)\right) .
\end{aligned}
$$

By Itô's formula we have,

$$
\begin{aligned}
d y^{u}(t)= & y^{u}(t)\left[\left\{\alpha b_{u}(t)+\frac{1}{2} \alpha^{2} \sigma_{u}^{2}(t)+\int_{\mathbb{R}}\left(\exp \left(\alpha \gamma_{u}(t, z)\right)-1-\alpha \gamma_{u}(t, z)\right) \nu(d z)\right\} d t\right. \\
& \left.+\alpha \sigma_{u}(t) d B(t)+\int_{\mathbb{R}}\left(\exp \left(\alpha \gamma_{u}(t, z)\right)-1\right) \tilde{N}(d t, d z)\right],
\end{aligned}
$$

where we have used the simplified notation

$$
b_{u}(t)=b(t, u(t), \omega), \quad \sigma_{u}(t)=\sigma(t, u(t), \omega) \gamma_{u}(t)=\gamma(t, u(t), \omega) .
$$

By the Itô martingale representation theorem for Lévy processes (see e.g. [15]) there exist predictable processes

$$
\varphi^{u}(t) \in L^{2}(\lambda \times P), \psi^{u}(t, z) \in L^{2}(\lambda \times P \times \nu)
$$

( $\lambda$ being Lebesgue measure on $[0, T]$ ) such that

$$
M^{u}(t)=M^{u}(0)+\int_{0}^{t} \varphi^{u}(s) d B(s)+\int_{0}^{t} \int_{\mathbb{R}} \psi^{u}(s, z) \tilde{N}(d s, d z)
$$


Combining (2.5) with (2.7) and (2.8) we get by the Itô product rule

$$
\begin{aligned}
d J^{u}(t)= & M^{u}(t) d y^{u}(t)+y^{u}(t) d M^{u}(t)+d\left[M^{u}, y^{u}\right](t) \\
= & J^{u}(t)\left[\left\{\alpha b_{u}(t)+\frac{1}{2} \alpha^{2} \sigma_{u}^{2}(t)+\int_{\mathbb{R}}\left(\exp \left(\alpha \gamma_{u}(t, z)\right)-1-\alpha \gamma_{u}(t, z)\right) \nu(d z)\right\} d t\right. \\
& \left.+\alpha \sigma_{u}(t) d B(t)+\int_{\mathbb{R}}\left(\exp \left(\alpha \gamma_{u}(t, z)\right)-1\right) \tilde{N}(d t, d z)\right] \\
& +y(t)\left[\varphi^{u}(t) d B(t)+\int_{\mathbb{R}} \psi^{u}(t, z) \tilde{N}(d t, d z)\right] \\
& +\alpha y^{u}(t) \sigma_{u}(t) \varphi^{u}(t) d t+y^{u}(t) \int_{\mathbb{R}}\left(\exp \left(\alpha \gamma_{u}(t, z)\right)-1\right) \psi^{u}(t, z) N(d t, d z) \\
= & \left\{J^{u}(t)\left[\alpha b_{u}(t)+\frac{1}{2} \alpha^{2} \sigma_{u}^{2}(t)+\int_{\mathbb{R}}\left(\exp \left(\alpha \gamma_{u}(t, z)\right)-1-\alpha \gamma_{u}(t, z)\right) \nu(d z)\right]\right. \\
& \left.+y^{u}(t)\left[\alpha \sigma_{u}(t) \varphi^{u}(t)+\int_{\mathbb{R}}\left(\exp \left(\alpha \gamma_{u}(t, z)\right)-1\right) \psi^{u}(t, z) \nu(d z)\right]\right\} d t \\
& +\left\{\alpha J^{u}(t) \sigma_{u}(t)+y^{u}(t) \varphi^{u}(t)\right\} d B(t) \\
& +\int_{\mathbb{R}}\left\{J^{u}(t)\left(\exp \left(\alpha \gamma_{u}(t, z)\right)-1\right)+y^{u}(t) \psi^{u}(t, z) \exp \left(\alpha \gamma_{u}(t, z)\right)\right\} \tilde{N}(d t, d z) .
\end{aligned}
$$

Now define

$$
Z^{u}(t)=\alpha J^{u}(t) \sigma_{u}(t)+y^{u}(t) \varphi^{u}(t)
$$

and

$$
K^{u}(t, z)=J^{u}(t)\left(\exp \left(\alpha \gamma_{u}(t, z)\right)-1\right)+y^{u}(t) \psi^{u}(t, z) \exp \left(\alpha \gamma_{u}(t, z)\right)
$$

Then

$$
\varphi^{u}(t)=\frac{1}{y^{u}(t)}\left[Z^{u}(t)-\alpha J^{u}(t) \sigma_{u}(t)\right]
$$

and

$$
\psi^{u}(t, z)=\frac{K^{u}(t, z)-J^{u}(t)\left(\exp \left(\alpha \gamma_{u}(t, z)\right)-1\right)}{y^{u}(t) \exp \left(\alpha \gamma_{u}(t, z)\right)} .
$$

Substituting (2.12) and (2.13) into (2.9) we get the following BSDE in the process $J^{u}(t)$

$$
\left\{\begin{array}{l}
d J^{u}(t)=-f\left(t, J^{u}(t), Z^{u}(t), K^{u}(t, \cdot), u(t)\right) d t+Z^{u}(t) d B(t)+\int_{\mathbb{R}} K^{u}(t, z) \tilde{N}(d t, d z) ; t \in[0, T] \\
J^{u}(T)=-\exp (-\alpha x-\alpha F)
\end{array}\right.
$$


where

$$
\begin{aligned}
f(t, y, z, k(\cdot), u(t))= & -y\left[\alpha b_{u}(t)+\frac{1}{2} \alpha^{2} \sigma_{u}{ }^{2}(t)+\int_{\mathbb{R}}\left(\exp \left(\alpha \gamma_{u}(t, z)\right)-1-\alpha \gamma_{u}(t, z)\right) \nu(d z)\right] \\
& -\alpha \sigma_{u}(t) z+\alpha^{2} \sigma_{u}^{2}(t) y-\int_{\mathbb{R}} \frac{\left(\exp \left(\alpha \gamma_{u}(t, z)\right)-1\right) k(z)}{\exp \left(\alpha \gamma_{u}(t, z)\right)} \nu(d z) \\
& +y \int_{\mathbb{R}} \frac{(\exp (\alpha \gamma(t, z))-1)^{2}}{\exp (\alpha \gamma(t, z))} \nu(d z) \\
= & -y\left[\alpha b_{u}(t)-\frac{1}{2} \alpha^{2} \sigma_{u}^{2}(t)+\int_{\mathbb{R}}\left\{1-\alpha \gamma_{u}(t, z)-\exp \left(-\alpha \gamma_{u}(t, z)\right)\right\} \nu(d z)\right] \\
& -\alpha \sigma_{u}(t) z-\int_{\mathbb{R}}\left(1-\exp \left(-\alpha \gamma_{u}(t, z)\right)\right) k(z) \nu(d z) .
\end{aligned}
$$

Theorem 2.1 (A BSDE approach to optimal control) Suppose that for all $(t, y, z, k(\cdot), \omega) \in[0, T] \times \mathbb{R} \times$ $\mathbb{R} \times \mathcal{R} \times \Omega$ there exists $\hat{u}(t)=\hat{u}(t, y, z, k(\cdot), \omega)$ such that

$$
f(t, y, z, k(\cdot), \hat{u}(t))=\underset{u}{e s s} \sup f(t, y, z, k(\cdot), u) .
$$

Suppose $\hat{u} \in \mathcal{A}$. Define the value process

$$
J(t)=\underset{u \in \mathcal{A}}{\operatorname{ess} \sup } J^{u}(t) .
$$

Suppose that for all $u \in \mathcal{A}$ there exists a unique solution $\left(Y^{u}(t), Z^{u}(t), K^{u}(t, \cdot)\right)$ of the BSDE

$$
\left\{\begin{array}{l}
d Y(t)=-f(t, Y(t), Z(t), K(t, \cdot), u(t)) d t+Z(t) d B(t)+\int_{\mathbb{R}} K(t, z) \tilde{N}(d t, d z) ; t \in[0, T] \\
Y(T)=-\exp (-\alpha x-\alpha F) .
\end{array}\right.
$$

Then $J(t)=Y^{\hat{u}}(t)$ for all $t \in[0, T]$. Moreover, the feedback control

$$
u^{*}(t):=\hat{u}(t, Y(t), Z(t), K(t, \cdot))
$$

is an optimal control for the problem (2.17).

Proof. Fix $u \in \mathcal{A}$ and let $\hat{u}$ be as in (2.16). Then

$$
\begin{aligned}
& f\left(t, J^{u}(t), Z^{u}(t), K^{u}(t, \cdot), \hat{u}\left(t, J^{u}(t), Z^{u}(t), K^{u}(t, \cdot)\right)\right) \\
\geq & f\left(t, J^{u}(t), Z^{u}(t), K^{u}(t, \cdot), u(t)\right) \quad \text { a.s. for all } t \in[0, T] .
\end{aligned}
$$

Define two drivers $f_{1}, f_{2}$ as follows:

$$
\begin{aligned}
& f_{1}(t, y, z, k(\cdot))=f(t, y, z, k(\cdot), \hat{u}(t, y, z, k(\cdot))), \\
& f_{2}(t, y, z, k(\cdot))=f(t, y, z, k(\cdot), u(t)) ; \quad t \in[0, T],
\end{aligned}
$$

and consider the two corresponding BSDEs

$$
\left\{\begin{array}{l}
d Y_{1}(t)=-f_{1}\left(t, Y_{1}(t), Z_{1}(t), K_{1}(t, \cdot)\right) d t+Z_{1}(t) d B(t)+\int_{\mathbb{R}} K_{1}(t, z) \tilde{N}(d t, d z) ; t \in[0, T] \\
Y_{1}(T)=-\exp (-\alpha x-\alpha F) .
\end{array}\right.
$$

$\mathrm{RR} \mathrm{n}^{\circ} 7554$ 


$$
\left\{\begin{array}{l}
d Y_{2}(t)=d J^{u}(t)=-f_{2}\left(t, J^{u}(t), Z^{u}(t), K^{u}(t, \cdot)\right) d t+Z^{u}(t) d B(t)+\int_{\mathbb{R}} K^{u}(t, z) \tilde{N}(d t, d z) ; t \in[0, T] \\
Y_{2}(T)=J^{u}(T)=-\exp (-\alpha x-\alpha F) .
\end{array}\right.
$$

Then, by 2.20)

$$
f_{1}\left(t, J^{u}(t), Z^{u}(t), K^{u}(t, \cdot)\right) \geq f_{2}\left(t, J^{u}(t), Z^{u}(t), K^{u}(t, \cdot)\right)
$$

and hence by the comparison theorem for BSDEs with jumps [21, we have

$$
Y_{1}(t) \geq Y_{2}(t)=J^{u}(t) \text { for all } t \in[0, T]
$$

In particular, if $u=\hat{u}$ we get $Y_{1}=J^{\hat{u}}(t)$ by uniqueness. Hence $\hat{u}=\hat{u}\left(t, J^{\hat{u}}(t), Z^{\hat{u}}(t), K^{\hat{u}}(t, \cdot)\right)$ is an optimal (feedback) control.

\section{Example 2.1 Optimal portfolio with exponential utility.}

Consider the following financial market:

- a risk free asset with unit price $S_{0}(t)=1 ; 0 \leq t \leq T$

- a risky asset, with unit price $S(t)$ given by

$$
d S(t)=S\left(t^{-}\right)\left[b_{0}(t) d t+\sigma_{0}(t) d B(t)+\int_{\mathbb{R}} \gamma_{0}(t, z) \tilde{N}(d t, d z)\right]
$$

where $b_{0}(t), \sigma_{0}(t)$ and $\gamma_{0}(t, z)$ are given $\mathcal{F}_{t}$-predictable processes such that $\gamma_{0} \geq-1+\epsilon$ for some $\epsilon>0$ and

$$
E\left[\int_{0}^{T}\left\{\left|b_{0}(t)\right|+\sigma_{0}^{2}(t)+\int_{\mathbb{R}} \gamma_{0}^{2}(t, z) \nu(d z)\right\} d t\right]<\infty
$$

If we let $u(t)$ denote a portfolio, representing the amount held in the risky asset at time $t$, then the dynamics of the value $X(t)=X^{u}(t)$ of the portfolio at time $t$ is

$$
\begin{aligned}
d X(t) & =u(t)\left[b_{0}(t) d t+\sigma_{0}(t) d B(t)+\int_{\mathbb{R}} \gamma_{0}(t, z) \tilde{N}(d t, d z)\right] \\
X(0) & =x \in \mathbb{R}
\end{aligned}
$$

Now consider the problem to find $u^{*} \in \mathcal{A}$ such that

$$
\sup _{u \in \mathcal{A}} E\left[-\exp \left(-\alpha X^{u}(T)-\alpha F\right)\right]=E\left[-\exp \left(-\alpha X^{u^{*}}(T)-\alpha F\right)\right]
$$

where $\mathcal{A}$ is the set of $\mathcal{F}_{t}$-adapted processes $u(t)$ such that

$$
E\left[\int_{0}^{T}\left\{\left|u(t) b_{0}(t)\right|+u^{2}(t) \sigma_{0}^{2}(t)+u^{2}(t) \int_{\mathbb{R}} \gamma_{0}^{2}(t, z) \nu(d z)\right\} d t\right]<\infty
$$

Comparing with 2.1 we see that in this case we have

$$
\begin{array}{r}
b(t, u(t))=u(t) b_{0}(t) \\
\sigma(t, u(t))=u(t) \sigma_{0}(t) \\
\gamma(t, u(t))=u(t) \gamma_{0}(t) .
\end{array}
$$

$\mathrm{RR} \mathrm{n}^{\circ} 7554$ 
Substituting this into (2.15) we get

$$
\begin{aligned}
& f\left(t, J^{u}(t), Z^{u}(t), K^{u}(t, \cdot), u(t)\right) \\
& =-J^{u}(t)\left[\alpha u(t) b_{0}(t)-\frac{1}{2} \alpha^{2} u^{2}(t) \sigma_{0}^{2}(t)+\int_{\mathbb{R}}\left\{1-\alpha u(t) \gamma_{0}(t, z)-\exp \left(-\alpha u(t) \gamma_{0}(t, z)\right)\right\} \nu(d z)\right] \\
& \quad-\alpha u(t) \sigma_{0}(t) Z^{u}(t)-\int_{\mathbb{R}}\left(1-\exp \left(-\alpha u(t) \gamma_{0}(t, z)\right)\right) K^{u}(t, z) \nu(d z) .
\end{aligned}
$$

Maximizing this with respect to $u$ gives the following first order condition for an optimal portfolio $\hat{u}(t)$ :

$$
\begin{array}{r}
-J(t)\left[b_{0}(t)-\alpha \sigma_{0}^{2}(t) \hat{u}(t)-\int_{\mathbb{R}} \gamma_{0}(t, z)\left(1+\exp \left(-\alpha \hat{u}(t) \gamma_{0}(t, z)\right)\right) \nu(d z)\right] \\
-\sigma_{0} Z(t)-\int_{\mathbb{R}} \gamma_{0}(t, z) \exp \left(-\alpha \hat{u}(t) \gamma_{0}(t, z)\right) K(t, z) \nu(d z)=0 .
\end{array}
$$

Thus we have proved

Corollary 2.2 Suppose there exists a unique solution $\hat{J}(t), \hat{Z}(t), \hat{K}(t, z)$ of the $B S D E$ (2.18), with $\hat{u}(t)=$ $\hat{u}(t, \hat{J}(t), \hat{Z}(t), \hat{K}(t, z))$ as in (2.26). Then $\hat{u}(t)$ is an optimal portfolio for the problem (2.23).

Case (i) : Consider the special case when $b_{0}(t), \sigma_{0}(t)$ and $\gamma_{0}(t, z)$ are deterministic. Then we can choose $\hat{Z}=\hat{K}=0$ in (2.18) and hence the equation (2.26) for $\hat{u}(t)$ reduces to

$$
b_{0}(t)-\alpha \sigma_{0}^{2}(t) \hat{u}(t)-\int_{\mathbb{R}} \gamma_{0}(t, z)\left(1+\exp \left(-\alpha \hat{u}(t) \gamma_{0}(t, z)\right)\right) \nu(d z)=0 .
$$

This result could also be obtained by dynamic programming.

Case (ii) : Consider the special case when there are no jumps, i.e. $\nu=0$. Then $\hat{u}(t)$ is found as

$$
\hat{u}(t)=\frac{b_{0}(t)}{\alpha \sigma_{0}^{2}(t)}+\frac{\hat{Z}(t)}{\alpha \sigma_{0}(t) \hat{J}(t)}
$$

where $\hat{J}(t), \hat{Z}(t)$ is the solution of the BSDE

$$
\left\{\begin{array}{l}
d \hat{J}(t)=\left(-\hat{J}(t)\left[\alpha \hat{u}(t) b_{0}(t)-\frac{1}{2} \alpha^{2} \hat{u}^{2}(t) \sigma_{0}^{2}(t)\right]-\alpha \hat{u}(t) \sigma_{0}(t) \hat{Z}(t)\right) d t+\hat{Z}(t) d B(t) ; t \in[0, T] \\
\hat{J}(T)=-\exp (-\alpha x-\alpha F)
\end{array}\right.
$$

i.e., using (2.27),

$$
\left\{\begin{array}{l}
d \hat{J}(t)=-\left[\frac{b_{0}^{2}(t) \hat{J}(t)}{2 \sigma_{0}^{2}(t)}+\frac{b_{0}(t) \hat{Z}(t)}{\sigma_{0}(t)}+\frac{\hat{Z}^{2}(t)}{2 \hat{J}(t)}\right] d t+\hat{Z}(t) d B(t) ; ; t \in[0, T] \\
\hat{J}(T)=-\exp (-\alpha x-\alpha F) .
\end{array}\right.
$$

Hence we get

Corollary 2.3 Suppose $\nu=0$ and there exists a unique solution $\hat{J}(t), \hat{Z}(t)$ of the BSDE (2.28). Then $\hat{u}(t)$ given by (2.27) is an optimal portfolio for the problem (2.23).

$\mathrm{RR} \mathrm{n}^{\circ} 7554$ 


\subsection{The power utility case}

Similarly, in the power utility case, with

$$
U(x)=\frac{1}{p} x^{p} ; x \in[0, \infty), \text { for some constant } p \in(-\infty, 1) \backslash\{0\}
$$

we study the problem to maximize

$$
F_{p}(u)=E\left[\frac{1}{p}\left(X^{u}(T)\right)^{p}\right]
$$

where

$$
\begin{aligned}
d X^{u}(t) & =X^{u}\left(t^{-}\right) u(t)\left[b_{0}(t) d t+\sigma_{0}(t) d B(t)+\int_{\mathbb{R}} \gamma_{0}(t, z) \tilde{N}(d t, d z)\right] \\
X^{u}(0) & =x>0 .
\end{aligned}
$$

In this case the control process $u(t)$ represents the fraction of the total wealth $X^{u}(t)$ invested in the risky asset, in the market given by (2.21). Then, again by the Itô formula,

$$
\begin{aligned}
X^{u}(T) & =x \exp \left(\int_{0}^{T} \sigma_{0}(s) u(s) d B(s)+\int_{0}^{T}\left\{b_{0}(s) u(s)-\frac{1}{2} \sigma_{0}^{2}(s) u^{2}(s)\right\} d s\right. \\
& +\int_{0}^{T} \int_{\mathbb{R}}\left\{\ln \left(1+u(s) \gamma_{0}(s, z)\right)-u(s) \gamma_{0}(s, z)\right\} \nu(d z) d s \\
& \left.+\int_{0}^{T} \int_{\mathbb{R}} \ln \left(1+u(s) \gamma_{0}(s, z)\right) \tilde{N}(d s, d z)\right) .
\end{aligned}
$$

Put

$$
\begin{aligned}
x_{t}^{u}(s) & =\int_{t}^{s} \sigma_{0}(r) u(r) d B(r)+\int_{t}^{s}\left(b_{0}(r) u(r)-\frac{1}{2} \sigma_{0}^{2}(r) u^{2}(r)\right) d r \\
& +\int_{t}^{s} \int_{\mathbb{R}}\left\{\ln \left(1+u(r) \gamma_{0}(r, z)\right)-u(r) \gamma_{0}(r, z)\right\} \nu(d z) d r \\
& +\int_{t}^{s} \int_{\mathbb{R}} \ln \left(1+u(r) \gamma_{0}(r, z)\right) \tilde{N}(d r, d z) ; 0 \leq t \leq s \leq T .
\end{aligned}
$$

We now define

$$
J^{u}(t)=E\left[\frac{1}{p}\left(x \exp \left(x_{t}^{u}(T)\right)\right)^{p} \mid \mathcal{F}_{t}\right] ; 0 \leq t \leq T .
$$

Then

$$
\begin{aligned}
J^{u}(t) & =\frac{x^{p}}{p} E\left[\exp \left\{p\left(x_{0}^{u}(T)-x_{0}^{u}(t)\right)\right\} \mid \mathcal{F}_{t}\right] \\
& =M(t) y(t)
\end{aligned}
$$

where

$$
M(t)=\frac{x^{p}}{p} E\left[\exp \left\{p x_{0}^{u}(T)\right\} \mid \mathcal{F}_{t}\right] \text { is a martingale }
$$

$\mathrm{RR} \mathrm{n}^{\circ} 7554$ 
and

$$
y(t)=\exp \left\{-p x_{0}^{u}(t\} \text { is } \mathcal{F}_{t}\right. \text {-adapted }
$$

Note that

$$
J^{u}(0)=F_{p}(u)
$$

and

Now we can proceed as in Section 2.1

$$
J^{u}(T)=\frac{x^{p}}{p}
$$

\subsection{The logarithmic utility case}

We consider now the logarithmic utility case, with

$$
U(x)=\ln x ; \quad x \in(0, \infty) .
$$

Then the problem is to maximize

$$
F_{0}(u):=E\left[\ln X^{u}(T)\right],
$$

where $X^{u}(t)$ is as in (2.31). Let $x_{t}^{u}$ be as in (2.33) and define

$$
J^{u}(t)=\ln x+E\left[x_{t}^{u}(T) \mid \mathcal{F}_{t}\right], \quad t \in[0, T] .
$$

Then

$$
J^{u}(0)=F_{0}(u) \text { and } J^{u}(T)=\ln x,
$$

and, since

$$
\ln x+x_{t}^{u}(T)=\ln x+x_{0}^{u}(T)-x_{0}^{u}(t)=\ln X(T)-x_{0}^{u}(t),
$$

we see that

$$
J^{u}(t)=M(t)-y(t)
$$

where

$$
M(t)=E\left[\ln X^{u}(T) \mid \mathcal{F}_{t}\right], y(t)=x_{0}^{u}(t) .
$$

Then by the martingale representation theorem we can write

$$
d M(t)=\varphi(t) d B(t)+\int_{\mathbb{R}_{0}} \psi(t, z) \tilde{N}(d t, d z)
$$

for some $\mathcal{F}_{t}$-adapted processes $\varphi(t), \psi(t)$. Then by the Itô formula,

$$
\begin{array}{r}
d J^{u}(t)=\varphi(t) d B(t)+\int_{\mathbb{R}_{0}} \psi(t, z) \tilde{N}(d t, d z)-\sigma_{0}(t) u(t) d B(t) \\
-\left(b_{0}(t) u(t)-\frac{1}{2} \sigma_{0}^{2}(t) u^{2}(t)\right) d t-\int_{\mathbb{R}_{0}}\left\{\ln \left(1+u(t) \gamma_{0}(t, z)\right)-u(t) \gamma_{0}(t, z)\right\} \nu(d z) d t \\
=\left\{-\int_{\mathbb{R}_{0}} \ln \left(1+u(t) \gamma_{0}(t, z)\right) \tilde{N}(d t, d z)\right. \\
+\left\{\varphi(t)-\sigma_{0}(t) u(t)\right\} d B(t)+\int_{\mathbb{R}_{0}}\left\{\psi(t, z)-\ln \left(1+u(t) \gamma_{0}(t, z)\right)\right\} \tilde{N}(d t, d z) .
\end{array}
$$

$\mathrm{RR} \mathrm{n}^{\circ} 7554$ 
Define

$$
Z^{u}(t)=\varphi(t)-\sigma_{0}(t) u(t)
$$

and

$$
K^{u}(t, z)=\psi(t, z)-\ln \left(1+u(t) \gamma_{0}(t, z)\right) .
$$

Substituting (2.43)-(2.44) into (2.42) we get the following BSDE for the process $J^{u}(t)$ :

$$
\begin{aligned}
d J^{u}(t) & =-f\left(t, J^{u}(t), Z^{u}(t), K^{u}(t, \cdot), u(t)\right) d t+Z^{u}(t) d B(t)+\int_{\mathbb{R}_{0}} K^{u}(t, z) \tilde{N}(d t, d z) ; t \in[0, T] \\
J^{u}(T) & =\ln x
\end{aligned}
$$

where

$$
f\left(t, J^{u}(t), Z^{u}(t), K^{u}(t, \cdot), u(t)\right)=b_{0}(t) u(t)-\frac{1}{2} \sigma_{0}^{2}(t) u^{2}(t)-\int_{\mathbb{R}_{0}}\left\{\ln \left(1+u(t) \gamma_{0}(t, z)\right)-u(t) \gamma_{0}(t, z)\right\} \nu(d z) .
$$

In this case we see that the maximizer $\hat{u}(t)$ of the driver $f$ is given by the equation

$$
b_{0}(t)-\sigma_{0}^{2}(t) \hat{u}(t)+\int_{\mathbb{R}_{0}} \frac{\hat{u}(t) \gamma_{0}^{2}(t, z)}{1+\hat{u}(t) \gamma_{0}(t, z)} \nu(d z)=0 .
$$

We conclude that this portfolio $\hat{u}(t)$ is optimal for the maximization of $F_{0}(u)=J^{u}(0)$. This is a well-known result which can be obtained by other methods as well. See e.g. [11.

\subsection{The general utility case}

So far we have been handling basically only the exponential utility case. In addition we have shown that the power utility and logarithmic utility cases can also be put into this framework under special assumptions on the dynamics of $X(t)$. It is of interest to be able to deal with general utility functions. We use here a different approach based on a stochastic maximum principle.

We restrict ourselves to the case without jumps, that is $\gamma=0$ in (2.1), so that the state equation is

$$
d X^{u}(t)=b(t, u(t)) d t+\sigma(t, u(t)) d B(t) ; X^{u}(0)=x
$$

where $u$ is some control process with values in $A$. We consider the following performance to maximize:

$$
J^{u}(0)=E\left[U\left(X^{u}(T)+F\right)\right]
$$

where $U$ is some general $C^{1}$ utility function and $F$ is a given bounded $\mathcal{F}_{T}$-measurable random variable.

We define the Hamiltonian

$$
H:[0, T] \times \mathbb{R} \times A \times \mathbb{R} \times \mathbb{R} \rightarrow \mathbb{R}
$$

by

$$
H(t, x, u, p, q)=b(t, u) p+\sigma(t, u) q,
$$

and the BSDE for the adjoint processes $p, q$ by:

$$
\begin{aligned}
d p^{u}(t) & =q^{u}(t) d B(t) ; 0 \leq t<T ; \\
p^{u}(T) & =U^{\prime}\left(X^{u}(T)+F\right) .
\end{aligned}
$$

$\mathrm{RR} \mathrm{n}^{\circ} 7554$ 
By the generalized Clark-Ocone formula [1], the solution of this BSDE is given by

$$
\begin{aligned}
& p^{u}(t)=E\left[U^{\prime}\left(X^{u}(T)+F\right) \mid \mathcal{F}_{t}\right] \\
& q^{u}(t)=E\left[D_{t} U^{\prime}\left(X^{u}(T)+F\right) \mid \mathcal{F}_{t}\right]
\end{aligned}
$$

where $D_{t}$ denotes the (generalized) Malliavin derivative at $t$. The stochastic maximum principle implies that if $u=\hat{u}$ is optimal then $\frac{\partial H}{\partial u}(t, x, u, p, q)=0$ at $\hat{u}$ that is, (denoting $b^{\prime}=\frac{\partial b}{\partial u}$ and $\sigma^{\prime}=\frac{\partial \sigma}{\partial u}$ )

$$
b^{\prime}(t, \hat{u}(t)) E\left[R \mid \mathcal{F}_{t}\right]+\sigma^{\prime}(t, \hat{u}(t)) E\left[D_{t} R \mid \mathcal{F}_{t}\right]=0
$$

where

$$
R=U^{\prime}\left(X^{\hat{u}}(T)+F\right) .
$$

By Theorem A.1 in [16, the general solution of this equation is $R=R_{\beta}(T)$ where

$$
R_{\beta}(T)=\beta \exp \left[\int_{0}^{T} \lambda(s) d B(s)-\frac{1}{2} \int_{0}^{T} \lambda^{2}(s) d s\right] .
$$

Here $\beta$ is an arbitrary constant and

$$
\lambda(t)=-\frac{b^{\prime}(t, \hat{u}(t))}{\sigma^{\prime}(t, \hat{u}(t))}
$$

This implies that, with $\hat{X}=X^{\hat{u}}$,

$$
\hat{X}(T)+F=I\left(R_{\beta}(T)\right)
$$

where

$$
I(y)= \begin{cases}\left(U^{\prime}\right)^{-1}(y) & 0 \leq y \leq y_{0} \\ 0 & y>y_{0}\end{cases}
$$

where $y_{0}=\lim _{x \rightarrow 0^{+}} U^{\prime}(x)$.

Therefore if we define

$$
\hat{Z}(t)=\sigma(t, \hat{u}(t))
$$

then we see by (2.47) and 2.50 that $\hat{X}(t), \hat{Z}(t)$ solve the BSDE:

$$
\left\{\begin{array}{l}
d \hat{X}(t)=b\left(t, \sigma^{-1}(t, \hat{Z}(t))\right) d t+\hat{Z}(t) d B(t) ; 0 \leq t \leq T \\
\hat{X}(T)=I\left(R_{\beta}(T)\right)-F
\end{array}\right.
$$

where $\sigma^{-} 1(t, y)$ is the inverse of the function $x \rightarrow \sigma(t, x)$ (assuming that this exists).

In the special case when

$$
b(t, u(t))=b_{0}(t) u(t) ; \quad \sigma(t, u(t))=\sigma_{0}(t) u(t),
$$

with $\sigma_{0}(t) \neq 0$, equation (2.52) becomes

$$
\left\{\begin{array}{l}
d \hat{X}(t)=\frac{b_{0}(t)}{\sigma_{0}(t)} \hat{Z}(t) d t+\hat{Z}(t) d B(t) ; 0 \leq t \leq T \\
\hat{X}(T)=I\left(R_{\beta}(T)\right)-F
\end{array}\right.
$$

with

$$
\hat{Z}(t)=\sigma_{0}(t) \hat{u}(t)
$$

RR $\mathrm{n}^{\circ} 7554$ 
In this case, $R_{\beta}(T)$ reduces to

$$
R_{\beta}(T)=\beta \exp \left[\int_{0}^{T}-\frac{b_{0}(t)}{\sigma_{0}(t)} d B(s)--\frac{1}{2} \int_{0}^{T}\left(\frac{b_{0}(s)}{\sigma_{0}(s)}\right)^{2}(s) d s\right] .
$$

The solution of the linear BSDE (2.53) is

$$
\hat{X}(t)=E\left[I\left(\left\{R_{\beta}(T)\right)-F\right\} \frac{R_{1}(T)}{R_{1}(t)} \mid \mathcal{F}_{t}\right] .
$$

In particular, choosing $t=0$ we get

$$
x=E\left[\left\{I\left(R_{\beta}(T)\right)-F\right\} R_{1}(T)\right],
$$

which is an equation which determines $\beta$.

With $\beta$ determined, $(\hat{X}(t), \hat{Z}(t))$ is determined by (2.53) and hence the optimal control $\hat{u}(t)$ is determined by (2.54), i.e.

$$
\hat{u}(t)=\frac{\hat{Z}(t)}{\sigma_{0}(t)}=\frac{D_{t} \hat{X}(t)}{\sigma_{0}(t)} .
$$

Using this, we see that the solution of (2.28) for $\hat{J}$ in the case of exponential utility is given by

$$
\hat{J}(t)=E\left[-\exp \left(-\alpha\left(x+\int_{t}^{T} b_{0}(s) \hat{u}(s) d s+\int_{t}^{T} \sigma_{0}(s) \hat{u}(s) d B_{s}\right)\right) \mid \mathcal{F}_{t}\right] .
$$

A further analysis in this direction with more general dynamics for the state process $X^{u}(t)$ is given in a companion paper [19] which addresses this issue by a forward-backward SDE games approach.

\section{$3 \quad$ BSDE games and application to portfolio optimization under model uncertainty}

In this section we assume that the control $u$ has 2 components, i.e.

$$
u(t)=(\pi(t), \theta(t))
$$

and we consider the stochastic differential game to find $\pi^{*} \in \mathcal{A}_{1}, \theta^{*} \in \mathcal{A}_{2}$ and $J^{\pi^{*}, \theta^{*}}$ such that

$$
J^{*}(t):=J^{\pi^{*}, \theta^{*}}(t)=\underset{\pi \in \mathcal{A}_{1}}{\operatorname{ess} \sup }\left(\underset{\theta \in \mathcal{A}_{2}}{\operatorname{ess} \inf } J^{\pi, \theta}(t)\right)
$$

where $J^{\theta, \pi}(t)=J^{u}(t)$ is as in (2.4). Proceeding as in Theorem 2.1 we obtain

Theorem 3.1 (BSDE games). Suppose that for all $(t, y, z, k(\cdot), \omega) \in[0, T] \times \mathbb{R} \times \mathbb{R} \times \mathcal{R} \times \Omega$ there exist $\hat{\pi}(t)=\hat{\pi}(t, y, z, k(\cdot), \omega)$ and $\hat{\theta}(t)=\hat{\theta}(t, y, z, k(\cdot), \omega)$ such that for all $(\pi, \theta) \in \mathcal{A}_{1} \times \mathcal{A}_{2}$

$$
f(t, y, z, k(\cdot), \pi(t), \hat{\theta}(t)) \leq f(t, y, z, k(\cdot), \hat{\pi}(t), \hat{\theta}(t)) \leq f(t, y, z, k(\cdot), \hat{\pi}(t), \theta(t)) \quad \text { for a.a. }(t, \omega) \text {. }
$$


where $f$ is as in 2.15). Suppose $\hat{\pi} \in \mathcal{A}_{1}$ and $\hat{\theta} \in \mathcal{A}_{2}$. Suppose that for all $u=(\pi, \theta) \in \mathcal{A}$ there exist unique solutions $\left(Y_{i}^{u}(t), Z_{i}^{u}(t), K_{i}^{u}(t, \cdot)\right)$ of the BSDEs

$$
\left\{\begin{array}{l}
d Y_{i}(t)=-f_{i}\left(t, Y_{i}(t), Z_{i}(t), K_{i}(t, \cdot)\right) d t+Z_{i}(t) d B(t)+\int_{\mathbb{R}} K_{i}(t, z) \tilde{N}(d t, d z) ; t \in[0, T] \\
Y_{i}(T)=-\exp (-\alpha x-\alpha F), \text { for } i=1,2,3
\end{array}\right.
$$

where

$$
\begin{aligned}
& f_{1}(t, y, z, k(\cdot))=f(t, y, z, k(\cdot), \pi(t), \hat{\theta}(t, y, z, k(\cdot))) \\
& f_{2}(t, y, z, k(\cdot))=f(t, y, z, k(\cdot), \pi(t), \theta(t)) \\
& f_{3}(t, y, z, k(\cdot))=f(t, y, z, k(\cdot), \hat{\pi}(t, y, z, k(\cdot)), \theta(t))
\end{aligned}
$$

Then

$$
\left.J^{\hat{\pi}, \hat{\theta}}(t)=\underset{\pi}{e s s \sup } J^{\pi, \hat{\theta}}(t)=J^{*}(t)=\underset{\theta}{\operatorname{essinf}} \underset{\theta}{\operatorname{ess} \sup } J^{\pi, \theta}(t)\right)=\underset{\theta}{\operatorname{essinf}} J^{\hat{\pi}, \theta}(t), t \in[0, T] .
$$

Moreover, $\pi^{*}(t):=\hat{\pi}(t, Y(t), Z(t), K(t, \cdot))$ and $\theta^{*}(t):=\hat{\theta}(t, Y(t), Z(t), K(t, \cdot))$ are optimal feedback controls, in the sense that they satisfy (3.1).

Proof. Since $f_{1} \leq f_{2} \leq f_{3}$ we have by the comparison theorem for BSDEs with jumps that

$$
J^{\pi, \hat{\theta}}(t)=Y_{1}(t) \leq Y_{2}(t)=J^{\pi, \theta}(t) \leq Y_{3}(t)=J^{\hat{\pi}, \theta}(t) .
$$

Since this holds for all $(\pi, \theta) \in \mathcal{A}_{1} \times \mathcal{A}_{2}$ we deduce that

$$
\begin{aligned}
& J^{\pi, \hat{\theta}}(t) \leq \underset{\theta}{\operatorname{essinf}} J^{\pi, \theta}(t), \text { for all } \pi \in \mathcal{A}_{1} \text {, } \\
& \text { ess sup } J^{\pi, \theta}(t) \leq J^{\hat{\pi}, \theta}(t) \text { for all } \theta \in \mathcal{A}_{2} .
\end{aligned}
$$

From the first of these inequalities we get

$$
\left.J^{\hat{\pi}, \hat{\theta}}(t) \leq \underset{\pi}{\operatorname{ess} \sup } J^{\pi, \hat{\theta}}(t) \leq \underset{\theta}{\mathrm{ess} \sup } \underset{\theta}{\text { essinf }} J^{\pi, \theta}(t)\right)=J^{*}(t)
$$

and from the second we get

$$
\left.\underset{\theta}{\operatorname{essinf}(\operatorname{ess} \sup } J^{\pi, \theta}(t)\right) \leq \underset{\theta}{\operatorname{essinf}} J^{\hat{\pi}, \theta}(t) \leq J^{\hat{\pi}, \hat{\theta}}(t) .
$$

Since we always have $\sup (\inf ) \leq \inf (\sup )$, we see that me must have equality everywhere in the last two chains of equalities. This proves (3.4) and hence completes the proof.

Remark 3.2 Condition (3.2) is equivalent to saying that the Isaacs condition holds, i.e.

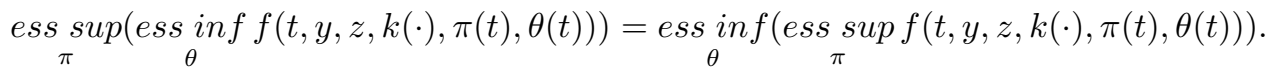

(See page 6 in [3] and the references therein).

$\mathrm{RR} \mathrm{n}^{\circ} 7554$ 


\section{Example 3.1 Portfolio optimization under model uncertainty.}

We now apply this to portfolio optimization under model uncertainty. We return to the market in Example 2.1. Let $V^{\pi}(t)$ be the wealth associated to the portfolio $\pi$, given by

$$
\begin{cases}d V^{\pi}(t) & =\pi(t)\left[b_{0}(t) d t+\sigma_{0}(t) d B(t)+\int_{\mathbb{R}} \gamma_{0}(t, z) \tilde{N}(d t, d z)\right] \\ V^{\pi}(0) & =x>0\end{cases}
$$

Here the control $\pi$ represents the amount invested in the risky asset at time $t$. We consider the additional feature of model uncertainty, represented by a probability measure $Q=Q^{\theta}$ which is equivalent to $P$, with the Radon-Nikodym derivative on $\mathcal{F}_{t}$ given by

$$
\frac{d\left(Q \mid \mathcal{F}_{t}\right)}{d\left(P \mid \mathcal{F}_{t}\right)}=G^{\theta}(t)
$$

where, for $0 \leq t \leq T, G^{\theta}(t)$ is a martingale of the form

$$
\begin{aligned}
d G^{\theta}(t) & =G^{\theta}\left(t^{-}\right)\left[\theta_{0}(t) d B(t)+\int_{\mathbb{R}} \theta_{1}(t, z) \tilde{N}(d t, d z)\right] \\
G^{\theta}(0) & =1 .
\end{aligned}
$$

Here $\theta=\left(\theta_{0}, \theta_{1}\right)$ may be regarded as a scenario control, assumed to be $\mathcal{F}_{t}$-predictable and such that $E\left[\int_{0}^{T}\left\{\left|\theta_{0}^{2}(t)\right|+\right.\right.$ $\left.\left.\int_{\mathbb{R}} \theta_{1}^{2}(t, z) \nu(d z)\right\} d t\right]<\infty$ and $\theta_{1}(t, z) \geq-1+\epsilon$ for some $\epsilon>0$.

Moreover, we introduce a kind of "penalty" for the scenario if its probability measure $Q^{\theta}$ deviates from the original measure $P$. This is in the spirit of [4, where an additive penalty in the form of entropy is studied. In our example the penalty is multiplicative and represented by a factor of the type

$$
\rho\left(\theta_{0}, \theta_{1}\right)=\exp \left(-\int_{0}^{T} g\left(\theta_{0}(s), \theta_{1}(s, \cdot)\right) d s\right),
$$

where $g: \mathbb{R} \times \mathcal{R} \rightarrow \mathbb{R}$ is a given function such that

$$
\int_{0}^{T}\left|g\left(\theta_{0}(s), \theta_{1}(s, \cdot)\right)\right| d s<\infty \text { for all } \theta \in \mathcal{A}_{2} .
$$

The factor (3.7) can also be seen as modeling the uncertainty on the instantaneous temporal preference rate. We assume that $g(\theta) \geq 0, g(0)=0$.

We consider now the performance functional

$$
L(u)=L(\pi, \theta):=E_{Q^{\theta}}\left[-\exp \left(-\alpha V^{\pi}(T)-\alpha F\right) \exp \left(-\int_{0}^{T} g(\theta(s)) d s\right)\right]
$$

where $F$ is a given bounded $\mathcal{F}_{T}$-measurable random variable. This is the model uncertainty aspect: the trader does not know what underlying probability measure $Q^{\theta}$ is used in the computation of the expected utility of the terminal wealth. She has to be prepared for the worst case scenario, and is thus led to the problem of finding $\pi^{*} \in \mathcal{A}_{1}, \theta^{*}=\left(\theta_{0}^{*}, \theta_{1}^{*}\right) \in \mathcal{A}_{2}$ such that

$$
\begin{array}{r}
\sup _{\pi \in \mathcal{A}_{1}}\left(\inf _{\theta \in \mathcal{A}_{2}} E_{Q^{\theta}}\left[-\exp \left(-\alpha V^{\pi}(T)-\alpha F-\int_{0}^{T} g(\theta(s)) d s\right)\right]\right) \\
=E_{Q^{\theta^{*}}}\left[-\exp \left(-\alpha V^{\pi^{*}}(T)-\alpha F-\int_{0}^{T} g\left(\theta^{*}(s)\right) d s\right)\right] .
\end{array}
$$

$\mathrm{RR} \mathrm{n}^{\circ} 7554$ 
This is a stochastic differential game of the type studied above. We note that

$$
L(\pi, \theta)=-E\left[G_{T}^{\theta} \exp \left(-\alpha V^{\pi}(T)-\alpha F-\int_{0}^{T} g(\theta(s)) d s\right)\right]
$$

where, by (3.6) and the Itô formula,

$$
\begin{aligned}
G^{\theta}(T) & =\exp \left(\int_{0}^{T} \theta_{0}(t) d B(t)-\frac{1}{2} \int_{0}^{T} \theta_{0}^{2}(t) d t+\int_{0}^{T} \int_{\mathbb{R}}\left\{\ln \left(1+\theta_{1}(t, z)\right)-\theta_{1}(t, z)\right\} \nu(d z) d t\right. \\
& +\int_{0}^{T} \int_{\mathbb{R}}\left\{\ln \left(1+\theta_{1}(t, z)\right) \tilde{N}(d t, d z)\right) .
\end{aligned}
$$

Hence, to be in the in the setup of Section 2.1] we define (with $u=(\pi, \theta)$ )

$$
X_{t}^{u}(s)=x+\int_{t}^{s} b(r, u(r)) d r+\int_{s}^{t} \sigma(r, u(r)) d B(r)+\int_{s}^{t} \int_{\mathbb{R}} \gamma(r, u(r), z) \tilde{N}(d r, d z)
$$

where

$$
\begin{aligned}
& b(t, u(t))=\frac{1}{2 \alpha} \theta_{0}^{2}(t)-\frac{1}{2} \sigma_{0}^{2}(t) \pi^{2}(t)+b_{0}(t) \pi(t)+\frac{1}{\alpha} g(\theta(t)) \\
& +\int_{\mathbb{R}}\left[-\frac{1}{\alpha} \ln \left(1+\theta_{1}(t, z)\right)+\frac{1}{\alpha} \theta_{1}(t, z)+\ln \left(1+\pi(t) \gamma_{0}(t, z)\right)-\pi(t) \gamma_{0}(t, z)\right] \nu(d z) \\
& \sigma(t, u(t))=-\frac{1}{\alpha} \theta_{0}(t)+\pi(t) \sigma_{0}(t) \\
& \gamma(t, u(t), z)=-\frac{1}{\alpha} \ln \left(1+\theta_{1}(t, z)\right)+\ln \left(1+\pi(t) \gamma_{0}(t, z)\right) .
\end{aligned}
$$

and

$$
J^{u}(t)=E\left[-\exp \left(-\alpha X_{t}^{u}(T)-\alpha F\right) \mid \mathcal{F}_{t}\right] ; 0 \leq t \leq T
$$

Then

$$
J^{u}(0)=L(\pi, \theta)
$$

and

$$
J^{u}(T)=-\exp (-\alpha(x-F)) .
$$

$J^{u}(t)$ now satisfies the BSDE

$$
\begin{aligned}
d J^{u}(t) & =-f\left(t, J^{u}(t), Z^{u}(t), K^{u}(t, \cdot), u(t)\right) d t+Z^{u}(t) d B_{t}+\int_{\mathbb{R}} K^{u}(t, z) \tilde{N}(d t, d z) ; t \in[0, T] \\
J^{u}(T) & =-\exp (-\alpha(x-F))
\end{aligned}
$$


where

$$
\begin{aligned}
f( & \left.t, J^{u}(t), Z^{u}(t), K^{u}(t, \cdot), u(t)\right) \\
= & -J^{u}(t)\left[-\frac{1}{2}\left(\alpha+\alpha^{2}\right) \sigma_{0}^{2}(t) \pi^{2}(t)+\alpha b_{0}(t) \pi(t)+\alpha \theta_{0}(t) \pi(t) \sigma_{0}(t)\right. \\
& +g\left(\theta_{0}(t), \theta_{1}(t, \cdot)\right) \\
& +\int_{\mathbb{R}}\left\{-\alpha \pi(t) \gamma_{0}(t, z)+\left(1+\theta_{1}(t, z)\right)\left(1-\left(1+\pi(t) \gamma_{0}(t, z)\right)^{-\alpha}\right) \nu(d z)\right] \\
+ & {\left[\theta_{0}(t)-\alpha \pi(t) \sigma_{0}(t)\right] Z^{u}(t) } \\
- & \int_{\mathbb{R}}\left\{\left(1-\left(1+\theta_{1}(t, z)\right)\left(1+\pi(t) \gamma_{0}(t, z)\right)^{-\alpha}\right\} K^{u}(t, z) \nu(d z) .\right.
\end{aligned}
$$

The first order condition for a maximum point $\pi=\hat{\pi}$ for $f$ is

$$
\begin{array}{r}
-J^{u}(t)\left[-(1+\alpha) \sigma_{0}^{2}(t) \hat{\pi}(t)+b_{0}(t)+\theta_{0}(t) \sigma_{0}(t)\right. \\
\left.+\int_{\mathbb{R}}\left\{-\gamma_{0}(t, z)+\left(1+\theta_{1}(t, z)\right)\left(1+\hat{\pi}(t) \gamma_{0}(t, z)\right)^{-\alpha-1} \gamma_{0}(t, z)\right\} \nu(d z)\right] \\
-\sigma_{0}(t) Z^{u}(t)-\int_{\mathbb{R}}\left(1+\theta_{1}(t, z)\right)\left(1+\hat{\pi}(t) \gamma_{0}(t, z)\right)^{-\alpha-1} \gamma_{0}(t, z) K^{u}(t, z) \nu(d z)=0
\end{array}
$$

The first order conditions for a minimum point $\left(\hat{\theta_{0}}, \hat{\theta_{1}}\right)$ for $f$ are

$$
\begin{aligned}
& -J^{u}(t)\left[\alpha \pi(t) \sigma_{0}(t)+\frac{\partial g}{\partial \theta_{0}}\left(\hat{\theta}_{0}, \hat{\theta}_{1}\right)\right]+Z^{u}(t)=0 \\
& -J^{u}(t)\left[\nabla_{\theta_{1}} g(\hat{\theta}(t))+\int_{\mathbb{R}}\left(1-\left(1+\pi(t) \gamma_{0}(t, z)\right)^{-\alpha}\right) \nu(d z)\right]+\int_{\mathbb{R}}\left(1+\pi(t) \gamma_{0}(t, z)\right)^{-\alpha} K^{u}(t, z) \nu(d z)=0 .
\end{aligned}
$$

The system (3.17), (3.18), (3.19) is hard to solve explicitly for $\hat{\pi}, \hat{\theta}_{0}$ and $\hat{\theta}_{1}$ in general. Let us consider some special cases.

Case (i) : Consider the special case when $b_{0}(t), \sigma_{0}(t)$ and $\gamma_{0}(t, z)$ are deterministic. Then we can choose $\hat{Z}=\hat{K}=0$ in (3.14) and hence the equations (3.17), (3.18), (3.19) reduce to

$$
\begin{aligned}
& b_{0}(t)+\sigma_{0}(t) \theta_{0}(t)-(1+\alpha) \sigma_{0}^{2}(t) \hat{\pi}(t)-\int_{\mathbb{R}} \gamma_{0}(t, z)\left(1-\left(1+\theta_{1}(t, z)\right)\left(1+\hat{\pi} \gamma_{0}(t, z)\right)^{-\alpha-1}\right) \nu(d z)=0 \\
& \alpha \pi(t) \sigma_{0}(t)+\frac{\partial g}{\partial \theta_{0}}\left(\hat{\theta}_{0}, \hat{\theta}_{1}\right)=0 \\
& \nabla_{\theta_{1}} g(\hat{\theta}(t))+\int_{\mathbb{R}}\left(1-\left(1+\pi(t) \gamma_{0}(t, z)\right)^{-\alpha}\right) \nu(d z)=0
\end{aligned}
$$

This result could also be obtained by dynamic programming. Indeed, the process $X_{t}^{\pi, \theta}$ is a Markovian process with generator

$$
\begin{array}{r}
A^{\pi, \theta} \Phi(t, x)=b(t, u(t)) \frac{\partial \Phi}{\partial x}(t, x)+\frac{1}{2} \sigma^{2}(t, u(t)) \frac{\partial^{2} \Phi}{\partial x^{2}}(t, x) \\
+\int_{\mathbb{R}}\left\{\Phi(t, x+\gamma(t, u, z))-\Phi(t, x)-\gamma(t, u, z) \frac{\partial \Phi}{\partial x}(t, x, z)\right\} \nu(d z) .
\end{array}
$$

RR $\mathrm{n}^{\circ} 7554$ 
If we define the value function

$$
\Phi(t, x)=\sup _{\pi \in \mathcal{A}_{1}} \inf _{\theta \in \mathcal{A}_{2}} E\left[-\exp \left(-\alpha X_{t}^{\pi, \theta}(T)-\alpha F\right)\right]
$$

then the Hamilton-Jacobi-Bellman-Isaacs equation for $\Phi$ is

$$
\begin{array}{r}
\frac{\partial \Phi}{\partial t}+\max _{\pi} \min _{\theta} A^{\pi, \theta} \Phi(t, x)=0 ; t \in[0, T[ \\
\Phi(T, x)=-\exp (-\alpha(x-F)) .
\end{array}
$$

Minimizing $A^{\pi, \theta} \Phi$ with respect to $\theta=\left(\theta_{0}, \theta_{1}(z)\right)$, and then maximizing $\pi \rightarrow A^{\pi, \hat{\theta}} \Phi$ with respect to $\pi$, and guessing that the value function is of the form $\Phi(t, x)=A(t) e^{-\alpha x}$ leads to the same first order conditions for an optimal $\hat{\pi}$ and optimal $\hat{\theta}$.

Case (ii) : Suppose now that $b_{0}(t), \sigma_{0}(t)$ are stochastic processes but consider the case when there are no jumps, i.e. $\nu=0$. Assume that $g(\theta)=g\left(\theta_{0}\right)$. then the system (3.17), 3.18), 3.19) reduces to

$$
\begin{aligned}
-J^{\hat{u}}(t)\left[-(1+\alpha) \sigma_{0}^{2}(t) \hat{\pi}(t)+b_{0}(t)+\hat{\theta}_{0}(t) \sigma_{0}(t)\right]-\sigma_{0}(t) Z^{\hat{u}}(t) & =0 \\
-J^{\hat{u}}(t)\left[\alpha \hat{\pi}(t) \sigma_{0}(t)+\frac{\partial g}{\partial \theta_{0}}\left(\hat{\theta}_{0}(t)\right)\right]+Z^{\hat{u}}(t) & =0 .
\end{aligned}
$$

In particular, if we assume that

$$
g\left(\theta_{0}\right)=\frac{\lambda}{2} \theta_{0}^{2}
$$

where $\lambda>0$, then the system (3.22)-(3.23) becomes linear in $\hat{\pi}, \hat{\theta}_{0}$, and we get the solution

$$
\begin{aligned}
\hat{\pi}(t) & =\frac{1}{\sigma_{0}(\alpha+\lambda(1+\alpha))}\left(\frac{\lambda b_{0}(t)}{\sigma_{0}}+(1+\lambda) \frac{Z^{\hat{u}}(t)}{J^{\hat{u}}(t)}\right) \\
\hat{\theta}_{0}(t) & =\frac{1}{\alpha+\lambda(1+\alpha)}\left(\frac{-\alpha b_{0}(t)}{\sigma_{0}(t)}+\frac{Z^{\hat{u}}(t)}{J^{\hat{u}}(t)}\right)
\end{aligned}
$$

where $J^{\hat{u}}(t), Z^{\hat{u}}(t)$ is the solution of the BSDE

$$
\left\{\begin{aligned}
d J^{\hat{u}}(t) & =\left\{J ^ { \hat { u } } ( t ) \left[-\frac{1}{2}\left(\alpha+\alpha^{2}\right) \sigma_{0}^{2}(t) \hat{\pi}^{2}(t)+\alpha b_{0}(t) \hat{\pi}(t)\right.\right. \\
& \left.\left.+\alpha \hat{\theta}_{0}(t) \hat{\pi}(t) \sigma_{0}(t)+\frac{1}{2} \lambda \hat{\theta}_{0}^{2}(t)\right]+\left[\hat{\theta}_{0}(t)-\alpha \hat{\pi}(t) \hat{\theta}_{0}(t)\right] Z^{\hat{u}}(t)\right\} d t+Z^{\hat{u}}(t) d B(t) ; t \in[0, T] \\
J^{\hat{u}}(T) & =-\exp (-\alpha(x-F))
\end{aligned}\right.
$$

Case (iii) : If $g(\theta)=0$ (no penalty), then all 3 first order conditions are satisfied if

$$
\hat{\pi}(t)=0, \quad Z^{\hat{u}}(t)=0, \quad K^{\hat{u}}(t, z)=0
$$

and if $\hat{\theta_{0}}, \hat{\theta_{1}}$ satisfy the equation

$$
b_{0}(t)+\hat{\theta_{0}}(t) \sigma_{0}(t)+\int_{\mathbb{R}} \hat{\theta_{1}}(t, z) \gamma_{0}(t, z) \nu(d z)=0 .
$$

$\mathrm{RR} \mathrm{n}^{\circ} 7554$ 
Condition (3.28) states that the measure $Q^{\hat{\pi}, \hat{\theta}}$ is an equivalent martingale measure for the price process $S(t)$ defined in (2.21). In this case the optimal strategy is to put all the money in the bank $\left(\pi^{*}=0\right)$. Since $J^{\hat{u}}(t)=-\exp (-\alpha x)<0$ for all $t \in[0, T]$, we see that $\hat{\pi}$ is a maximum point for $f$ and $\left(\hat{\theta_{0}}, \hat{\theta_{1}}\right)$ is a minimum point. This result had been proved before in [12, 17] in the Markovian case using HJB-Isaacs equations and in 18 in the general case by means of the maximum principle.

Acknowledgments. We thank Marie-Claire Quenez and Shige Peng for useful comments.

\section{References}

[1] K. Aase, B. Øksendal, N. Privault and J. Ubøe: White noise generalizations of the Clark-Haussmann-Ocone theorem, with applications to mathematical finance. Finance 8 Stochastics 4 (2000), 465-496.

[2] T.T.K. An and B. Øksendal: A maximum principle for stochastic differential games with $g$-expectation and partial information. Eprint, Dept of Math., Univ. of Oslo 4/2010. To appear in Stochastics.

[3] R.J. Elliott and T. K. Siu: A BSDE approach to a risk-based optimal investment of an insurer. Automatica (2010), doi:10.1016/j.automatica.2010.10.032

[4] G. Bordigoni, A. Matoussi and M. Schweizer: A stochastic control approach to a robust utility maximization problem. In F.E. Benth et al (editors): Stochastic Analysis and Applications. The Abel Symposium 2005. Springer 2007, pp. 125-151.

[5] X. De Scheemaekere: Dynamic risk indifference pricing in incomplete markets. Manuscript September 11, 2009.

[6] G. Di Nunno, T. Meyer-Brandis, B. Øksendal and F. Proske: Malliavin calculus and anticipative Itô formulae for Lévy processes. Inf. Dim. Anal. Analysis, Quantum Prob. and Related Topics 8 (2005), 235258.

[7] G. Di Nunno, B. Øksendal and F. Proske: Malliavin Calculus for Lévy Processes with Applications to Finance. Springer 2009.

[8] N. El Karoui, S. Peng and M.-C. Quenez: BSDEs in Finance Math. Finance, 1997, Vol.7, 1, 1-71.

[9] S. Hamadène: Backward-forward SDE's and stochastic differential games. Stochastic processes and their applications 77 (1998), 1-15.

[10] Y. Hu, P. Imkeller and M. Muller: Utility maximization in incomplete markets. Ann. Appl. Probab. 15 (2005), 1691-1712.

[11] T. Lim and M.-C. Quenez: Exponential utility maximization and indifference price in an incomplete market with defaults. Manuscript May 2010.

[12] M. Mania and M. Schweizer: Dynamic exponential utility indifference pricing valuation, The Annals of Applied Probability 15 (2005), 2113-2143.

[13] M. Mania and R. Tevzadze: Backward stochastic PDEs related to the utility maximization problem. ArXiv:0806.0240v, 2 June 2008.

RR $n^{\circ} 7554$ 
[14] S. Mataramvura and B. Øksendal: Risk minimizing portfolios and HJBI equations for stochastic differential games. Stochastics, 80(4), 317-337.

[15] B. Øksendal and A. Sulem: Applied Stochastic Control of Jump Diffusions. Second Edition, Springer 2007.

[16] B. Øksendal and A. Sulem: Maximum principles for optimal control of forward-backward stochastic differential equations with jumps. SIAM J. Control Optimization, (2009), Vol. 48,5, 2845-2976.

[17] B. Øksendal and A. Sulem: A game theoretic approach to martingale measures in incomplete markets. Eprint, Dept. of Mathematics, University of Oslo 24/2006. Survey of Applied and Industrial Mathematics (TVP Publishers, Moscow), 15, (2008), 18-24.

[18] B. Øksendal and A. Sulem: Robust stochastic control and equivalent martingale measures. Eprint, Dept of Mathematics, University of Oslo 8/2010.

[19] B. Øksendal and A. Sulem: Optimal strategies under model uncertainty for general utilities and forwardbackward SDE games, Manuscript 2011.

[20] H. Pham: Continuous -time Stochastic Control and Optimization with Financial Applications. Springer 2009.

[21] M. Royer : Backward stochastic differential equations with jumps and related non-linear expectations, Stochastic Processes and Their Applications 116 (2006), 1358-1376.

RR $\mathrm{n}^{\circ} 7554$ 


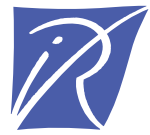

Centre de recherche INRIA Paris - Rocquencourt

Domaine de Voluceau - Rocquencourt - BP 105 - 78153 Le Chesnay Cedex (France)

Centre de recherche INRIA Bordeaux - Sud Ouest : Domaine Universitaire - 351, cours de la Libération - 33405 Talence Cedex

Centre de recherche INRIA Grenoble - Rhône-Alpes : 655, avenue de l'Europe - 38334 Montbonnot Saint-Ismier

Centre de recherche INRIA Lille - Nord Europe : Parc Scientifique de la Haute Borne - 40, avenue Halley - 59650 Villeneuve d'Ascq

Centre de recherche INRIA Nancy - Grand Est : LORIA, Technopôle de Nancy-Brabois - Campus scientifique

615, rue du Jardin Botanique - BP 101 - 54602 Villers-lès-Nancy Cedex

Centre de recherche INRIA Rennes - Bretagne Atlantique : IRISA, Campus universitaire de Beaulieu - 35042 Rennes Cedex

Centre de recherche INRIA Saclay - Île-de-France : Parc Orsay Université - ZAC des Vignes : 4, rue Jacques Monod - 91893 Orsay Cedex

Centre de recherche INRIA Sophia Antipolis - Méditerranée : 2004, route des Lucioles - BP 93 - 06902 Sophia Antipolis Cedex 